\title{
The Mixed Current Model of Nano-MOSFETs Considering the Effect of Horizontal and Vertical Electric Fields
}

\author{
Heng-Sheng Huang ${ }^{1}$, Ping-Ray Huang ${ }^{1}$, Mu-Chun Wang ${ }^{1,2, *}$, Shuang-Yuan \\ Chen $^{1}$, Shea-Jue Wang ${ }^{3}$, Ching-Chuan Chou ${ }^{2}$, LS Huang ${ }^{4}$, Wei-Lun Wang ${ }^{1}$
}

\footnotetext{
${ }^{1}$ Graduate Institute of Mechatronic Engineering, National Taipei University of Technology, Taipei 10608, Taiwan.

${ }^{2}$ Department of Electronic Engineering, Minghsin University of Science and Technology, Hsinchu 30401, Taiwan.

${ }^{3}$ Department of Materials and Resources Engineering, National Taipei University of Technology, Taipei 10608, Taiwan.

${ }^{4}$ Reliability Technology and Assurance Division, United Microelectronics Corporation, Hsinchu 30078, Taiwan.

*Correspondence author: mucwang@must.edu.tw
}

\begin{abstract}
A novel drive current model covering the effects of source/drain voltage (VDs) and gate voltage (VGs) and incorporating drift and diffusion current on the surface channel at the nano-node level, especially beyond $28 n m$ node is presented. The effect of the diffusion current added is more satisfactory to describe the behavior of the drive current in nano-node MOSFETs, fabricated with the atomic-layer-deposition (ALD) technology. This breakthrough in model establishment can expose the long and short channel devices together. Introducing the variables of $V_{D S}$ and $V_{G S}$, the mixed current model more effectively and meaningfully demonstrates the drive current of MOSFETs under the operation of horizontal, vertical, or mixed electrical field. In comparison between the simulation and experimental consequences, the electrical performance is impressive. The error between both is less than $1 \%$, better than the empirical adjustment to issue a set of drive current models.
\end{abstract}

Index Terms_-drift current, diffusion current, mobility, nano-node, model, ALD technology.

\section{INTRODUCTION}

In conventional device models for metal-oxide-semiconductor field-effect transistors (MOSFETs), the drive current [1,2] mainly considers the moving carriers with the drift effect. As the channel length of MOSFET keeps scaling down, the saturation current of MOSFETs raises up with the increase of the drain voltage or the decrease of the channel length. There are some published literatures to explain this phenomenon, such as channel length modulation [3-5] and velocity overshoot [6-9]. However, the proposed conduction mechanisms seem not fully to fit the physically measured current-voltage results well. To compensate this drawback, the device models in semiconductor foundries usually assist some empirical adjustment, but no physical meanings. Here, we propose a new concept incorporating the diffusion effect in each channel point due to the gradient of inversion charge density, especially near the pinch-off point. At this point, the inversion charge density traditionally approaches to zero, indicating the drift current zero, but the real drive current is not zero.

As a result, the drive current rises as the drain voltage increases for a nano-scale MOSFET. Adding the diffusion effect in drive-current model, the contribution of diffusion current in entire drive current is distinctly enhanced, especially in the current behavior of short channel devices [10]. In other words, a lager source/drain voltage $\mathrm{V}_{\mathrm{DS}}$ will make a lager gradient of inversion charge density causing a larger surface diffusion current. However, the previous work only changing one parameter $\left(V_{D S}\right)$ as $V_{G S}$ fixed in long or short channel devices to fit the equivalent mobility $\mu_{\text {eq }}$ accurately was successfully finished. In this work, we furthermore propose a deeper consideration incorporating the modulation of all the parameters $\left(\mathrm{V}_{\mathrm{DS}}, \mathrm{V}_{\mathrm{GS}}\right.$ and $\left.\mathrm{L}_{\text {mask }}\right)$ at one time

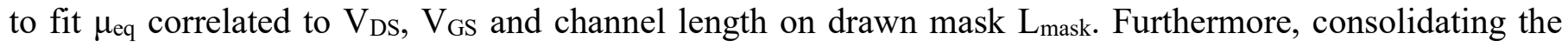
adjustment of $V_{D S}$ and $L_{\text {mask }}$ correlated to the horizontal electric field and $V_{\mathrm{GS}}$ influencing the vertical electric field, the drive current of MOSFETs is more meaningful and beneficial in providing a set of accurate nano- 
node device models, especially beyond $28 \mathrm{~nm}$ node. The gate dielectric in this work was the sandwich stack of $\mathrm{HfO}_{\mathrm{x}} / \mathrm{ZrO}_{\mathrm{y}} / \mathrm{HfO}_{z}$ deposited with the atomic layer deposition (ALD) technology. The physical thickness with ALD technology is about $24 \AA$

\section{II. EXPERIMENTAL AND FITTING THE MOBILITY}

Based on the previous work [10], the carrier mobility $\mu(\mathrm{x})$ should be the function of the position $\mathrm{x}$ in the surface channel. But it seems not enough to describe the whole behavior of an equivalent mobility $\mu_{\mathrm{eq}}$ correlated to $\mathrm{V}_{\mathrm{DS}}, \mathrm{V}_{\mathrm{GS}}$, and $\mathrm{L}_{\text {mask}}$, independent of the position $\mathrm{x}$. Incorporating the various conditions with $\mathrm{V}_{\mathrm{DS}}, \mathrm{V}_{\mathrm{GS}}$ and $\mathrm{L}_{\text {mask }}$ is to reasonably describe the whole drive current and fit the $\mu_{\mathrm{eq}}$ as shown in Eqs. (1)-(4):

$$
\begin{gathered}
I_{\text {Drift }}=Q_{i}(x) \times v_{d}(x) \times W \\
I_{\text {Diff }}=D_{n} \times \frac{d Q_{i}(x)}{d x} \times W \quad ; D_{n}=\mu_{e q} \times k T / q \\
\frac{I_{\text {total }}}{W}=I_{\text {drift }}+I_{\text {diff }}=-Q_{i}(x) \times v_{d}(x)+D_{n} \times \frac{d Q_{i}(x)}{d x} \\
\frac{I_{\text {total }}}{W}=-\frac{\mu_{e q} E_{s a t} Q_{i}(x) \frac{d Q_{i}(x)}{d x}}{m C_{o x} E_{s a t}+\frac{d Q_{i}(x)}{d x}}+D_{n} \times \frac{d Q_{i}(x)}{d x}
\end{gathered}
$$

where q: unit charge, $\mathrm{W}$ : channel width, $v_{\mathrm{d}}$ : carrier drift velocity, $D_{\mathrm{n}}$ : diffusion coefficient of electron carrier, $E_{\text {sat: }}$ horizontal electric field in saturation, k: Boltzmann constant, T: absolute temperature, and $m$ : body factor.

Under the specific conditions such as fixing a channel length and tuning the gate voltages to sense the IDS$\mathrm{V}_{\mathrm{DS}}$ characteristics, we can derive the relationship between the $\mu_{\mathrm{eq}}$ and the drain bias, as shown in Fig. 1. Through changing the fixed channel length and the drain bias, the linear and saturation regions are obviously represented no matter what the tested channel device is. It is important to seek the accurate relationship between these three parameters and the $\mu_{\text {eq. }}$. In this work, there are four sections $(a, b, c, d)$ illustrating longshort channel devices and linear-saturation regions split at $\mathrm{L}_{\text {mask }}=120 \mathrm{~nm}$ due to the classification of electrical measurement, as shown in Table I. 


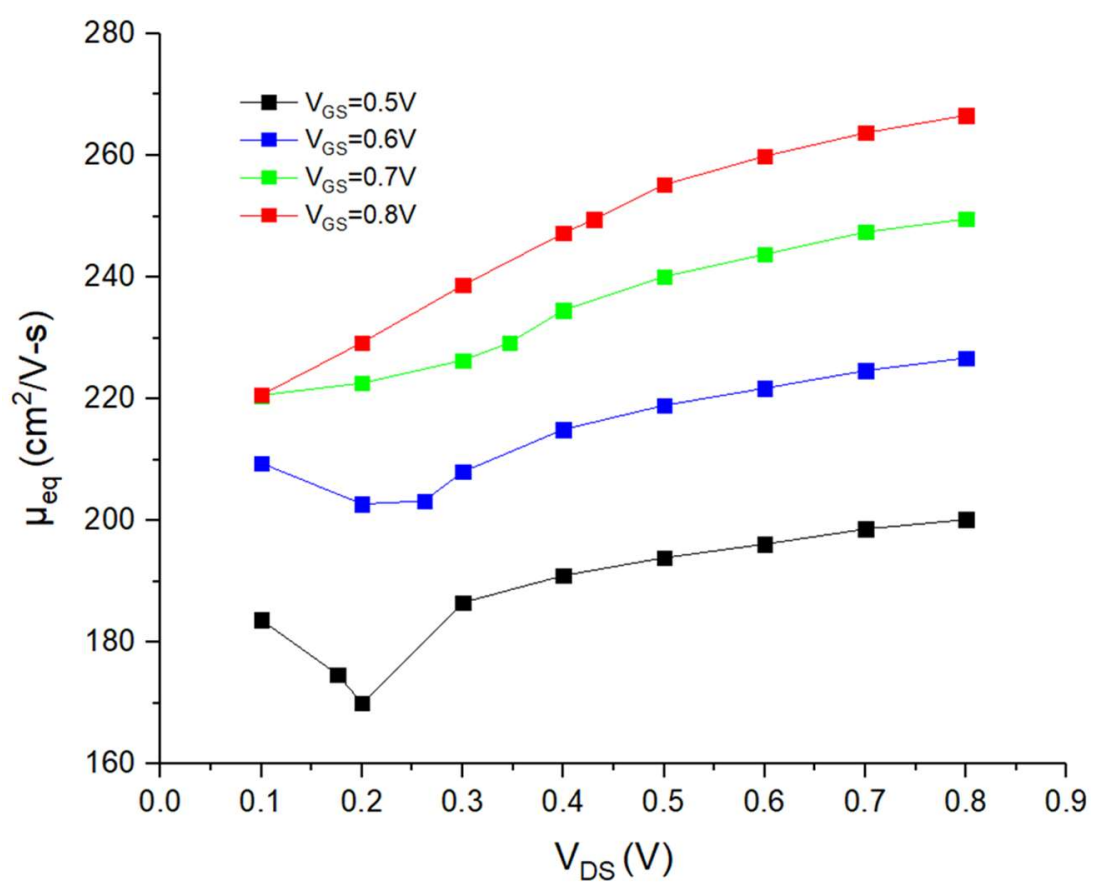

Fig. 1. $\mu_{\mathrm{eq}}$ Vs. $\mathrm{V}_{\mathrm{DS}}$ under different $\mathrm{V}_{\mathrm{GS}}$ at $\mathrm{L}_{\text {mask }}=500 \mathrm{~nm}$ in section a.

Table I. Four sections classified as long-short devices and linear-saturation regions.

\begin{tabular}{ll}
\hline Long channel $(>120 \mathrm{~nm})$, Linear region & $X=\mathrm{a}$ \\
Long channel $(>120 \mathrm{~nm})$, Saturation region & $X=\mathrm{b}$ \\
Short channel $(<120 \mathrm{~nm})$, Linear region & $X=\mathrm{c}$ \\
Short channel $(<120 \mathrm{~nm})$, Saturation region & $X=\mathrm{d}$ \\
\hline
\end{tabular}

First, the $\mu_{\text {eq }}$ can be written as a function of $V_{D S}$ in different $V_{G S}$ at fixed $L_{\text {mask, }}$ which can be described by the Taylor expansion until the third-order polynomial, as shown in Eq.(5):

$$
\mu_{e q}\left(V_{D S}\right)=\sum_{i=0}^{3} X_{i} \times\left(V_{D S}\right)^{i}
$$

where $X_{\mathrm{i}}$ is the coefficient of the $\mathrm{i}^{\text {th }}$ polynomial and $\mathrm{i}=0,1,2,3 . X$ can be attributed as each section $(\mathrm{a}, \mathrm{b}, \mathrm{c}$, or d).

Continuously, the $X_{\mathrm{i}}$ is strongly related to the gate voltages as $\mathrm{L}_{\text {mask }}$ fixed, which can be given as Eq.(6) with the Taylor expansion.

$$
X_{i}\left(V_{G S}\right)=\sum_{j=0}^{3} X_{i j} \times\left(V_{G S}\right)^{j}
$$

where $X_{\mathrm{ij}}$ is the coefficient of $\mathrm{V}_{\mathrm{GS}}$ polynomials with $\mathrm{j}=0,1,2,3$.

Ultimately, the impact factor $\mathrm{L}_{\text {mask }}$ to the coefficient $X_{\mathrm{ij}}$ is entirely represented as Eq.(7):

$$
X_{i j}\left(L_{\text {mask }}\right)=\sum_{k=0}^{2} X_{i j k} \times\left(L_{\text {mask }}\right)^{k}
$$

where $X_{\mathrm{ijk}}$ is the coefficient of $\mathrm{L}_{\text {mask }}$ variable with $\mathrm{k}=0,1,2$.

The flow chart of $X$-coefficients and the $\mu_{\text {eq }}$ value in extraction is demonstrated in Fig. 2 with section a as an example. The other sections in parameter extraction also follow the same procedures to achieve the accurate $\mu_{\mathrm{eq}}$ value correlated to $\mathrm{V}_{\mathrm{DS}}, \mathrm{V}_{\mathrm{GS}}$, and $\mathrm{L}_{\text {mask }}$ variables. Table II demonstrates the full relationship of parameters and $X$-coefficients. 
How to seek the coefficient values

Step 1. Take the section a as an
example, equations can all be
represented by the first function in
the graph of $\mu_{\mathrm{eq}}-\mathrm{V}_{\mathrm{DS}}, \mathrm{L}_{\text {mask }}=1 \mu \mathrm{m}$,
$0.5 \mu \mathrm{m}$ and $120 \mathrm{~nm}$.

Step 2. Under the same $\mathrm{L}_{\text {mask, }}$ different $\mathrm{V}_{\mathrm{GS}}$ will have different coefficient values, so that we find the relationship among $\mathrm{V}_{\mathrm{GS}}$ and the coefficient code $\left(\mathrm{a}_{3}\right)$. Then we can get the coefficient codes $\left(a_{30}, a_{31}\right.$, $\left.\mathrm{a}_{32}, \mathrm{a}_{33}\right)$
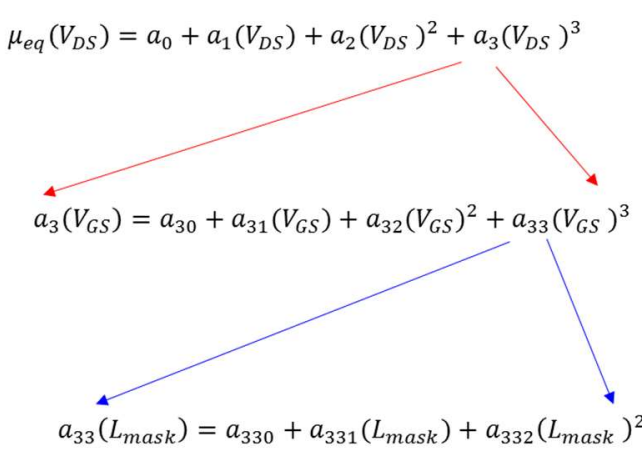

How to fit the $\mu_{\mathrm{eq}}$

Step III. Repeat steps I and II to get the values $\left(a_{0}, a_{1}, a_{2}\right)$ and introduce $\mathrm{V}_{\mathrm{DS}}$, we can fit the value of the $\mu_{\text {eq }}$.

Step II. With the coefficient values $\left(a_{30}, a_{31}, a_{32}, a_{33}\right)$, we can get the value $\left(\mathrm{a}_{3}\right)$ including $\mathrm{V}_{\mathrm{GS}}$.

Step I. When we get the coefficient values $\left(a_{300}, a_{301}, a_{302}, a_{310}, a_{311}, a_{312}\right.$, $\left.\mathrm{a}_{320}, \mathrm{a}_{321}, \mathrm{a}_{322}, \mathrm{a}_{330}, \mathrm{a}_{331}, \mathrm{a}_{332}\right)$, we can get back the values $\left(a_{30}, a_{31}, a_{32}, a_{33}\right)$ by substituting $\mathrm{L}_{\text {mask }}$.

between $L_{\text {mask }}$ and values of coefficient $a_{3 j}\left(a_{30}\right.$, $\left.a_{31}, a_{32}, a_{33}\right)$. Finally we can further get the values of coefficient $a_{3 j k}\left(a_{300}, a_{301}, a_{302}, a_{310}, a_{311}, a_{312}\right.$, $a_{320}, a_{321}, a_{322}, a_{330}, a_{331}, a_{332}$ ).

Fig. 2. The flow chart for extracting the coefficients and $\mu_{\text {eq }}$ values with section a as an example.

Table II. The relationship among each parameter and the coefficient in Taylor expansion.

The coefficient of $\mu_{\mathrm{eq}}\left(\mathrm{V}_{\mathrm{DS}}\right) \quad X_{\mathrm{i}}$ : the functions of $\mathrm{V}_{\mathrm{GS}}$, $\mathrm{i}=0,1,2,3$

The coefficient of $X_{\mathrm{i}}\left(\mathrm{V}_{\mathrm{GS}}\right) \quad X_{\mathrm{ij}}$ : the functions of Lmask, $\mathrm{j}=0,1,2,3$

The coefficient of $X_{\mathrm{ij}}\left(\mathrm{L}_{\text {mask }}\right) \quad X_{\mathrm{ijk}}$ : constant, $\mathrm{k}=0,1,2$

\section{III. RESULTS AND DISCUSSION}

Following the last flow chart to obtain the coefficients $\left(X_{\mathrm{i}}, X_{\mathrm{ij}}, X_{\mathrm{ijk}}\right)$, the $\mu_{\mathrm{eq}}\left(\mathrm{V}_{\mathrm{DS}}, \mathrm{V}_{\mathrm{GS}}, \mathrm{L}_{\text {mask }}\right)$ at any condition can be effectively and meaningfully extracted. After that, the equivalent mobility will be immediately substituted into (4) to do the simulation of the corresponding drive current. The simulation and experimental results of I-V curves with long and short channel devices represented as four sections are shown in Fig. 3. 

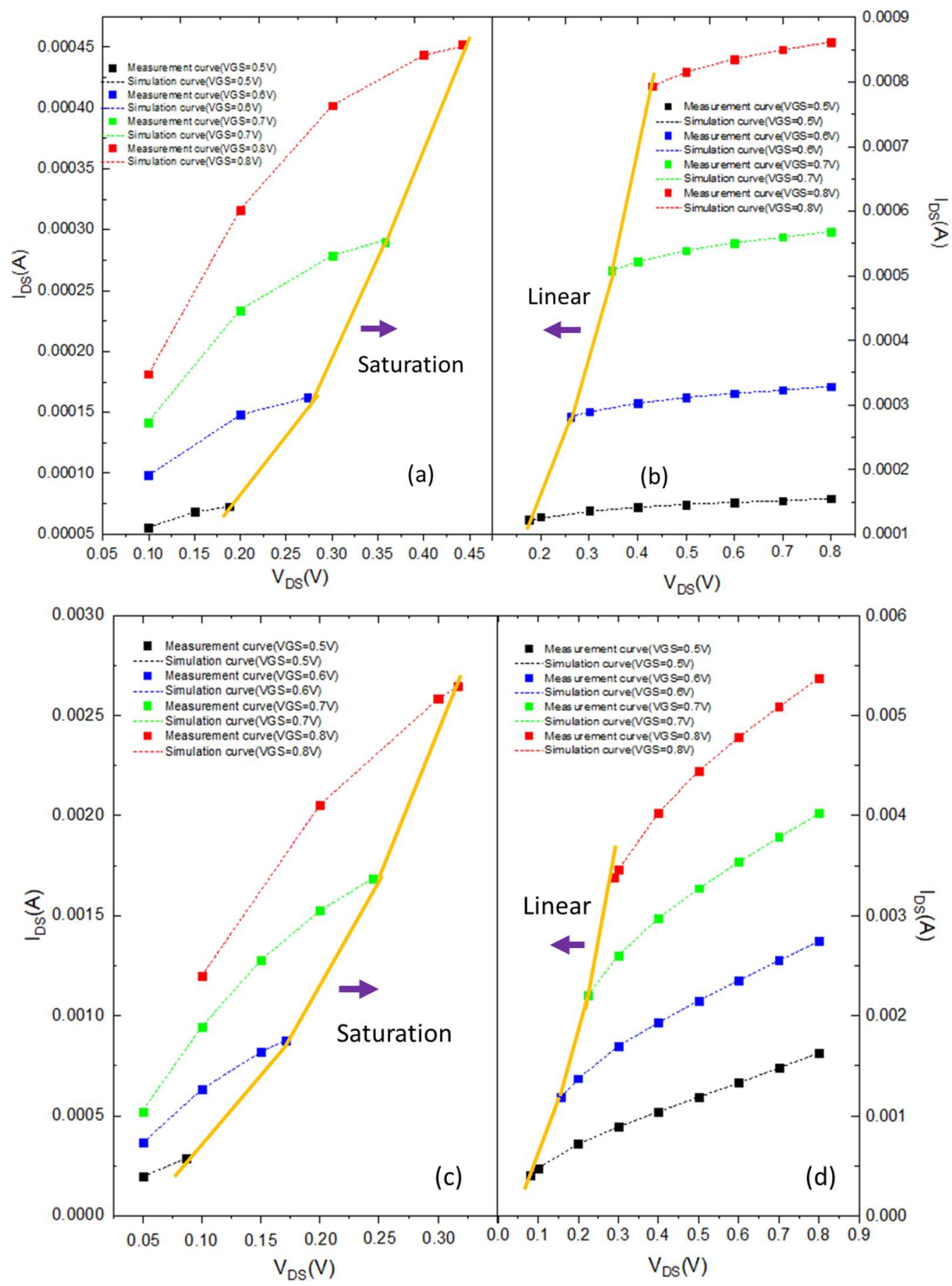

Fig. 3. $\mathrm{I}_{\mathrm{DS}}$ vs. $\mathrm{V}_{\mathrm{DS}}$ curves for long-channel devices: (a) at $\mathrm{L}_{\text {mask }}=1 \mu \mathrm{m}$ in section a and (b) at $\mathrm{L}_{\text {mask }}=500 \mathrm{~nm}$ in section b; for short-channel devices: (c) at $\mathrm{L}_{\text {mask }}=50 \mathrm{~nm}$ in section $\mathrm{c}$ and (d) at $\mathrm{L}_{\text {mask }}=33 \mathrm{~nm}$ in section d.

For precise observation of the contribution of the $\mu_{\text {eq }}$ correlated to $\mathrm{V}_{\mathrm{DS}}, \mathrm{V}_{\mathrm{GS}}$, and $\mathrm{L}_{\text {mask}}$, one 3-D plot is established and shown in Fig. 4, combining all of these extracted data linked as lines and extended these lines as a continuous plane.

In this study, the tested channel-length devices contain 33, 50,90, 120,500 nm and $1 \mu \mathrm{m}$. As the channel length $\mathrm{L}$ is greater than $120 \mathrm{~nm}$, the section is defined as the long-channel section. Thus, the plot in Fig.4 with $\mathrm{L}=500 \mathrm{~nm}$ is represented as long-channel section and with $\mathrm{L}=33 \mathrm{~nm}$ as short-channel one. Furthermore, the $\mathrm{V}_{\mathrm{DS}}$ and $\mathrm{V}_{\mathrm{GS}}$ sensed are $0.1,0.2 \ldots, 0.8 \mathrm{~V}$ and $0.5,0.6,0.7,0.8 \mathrm{~V}$, respectively. The error between simulation and real measurement data is less than $1 \%$ no matter what the channel-length devices and the 
operation mode at linear or saturation region are. Table III is an example to extract the coefficients in section a under $\mathrm{V}_{\mathrm{GS}}=0.8 \mathrm{~V}$ and $\mathrm{L}_{\text {mask }}=500 \mathrm{~nm}$.

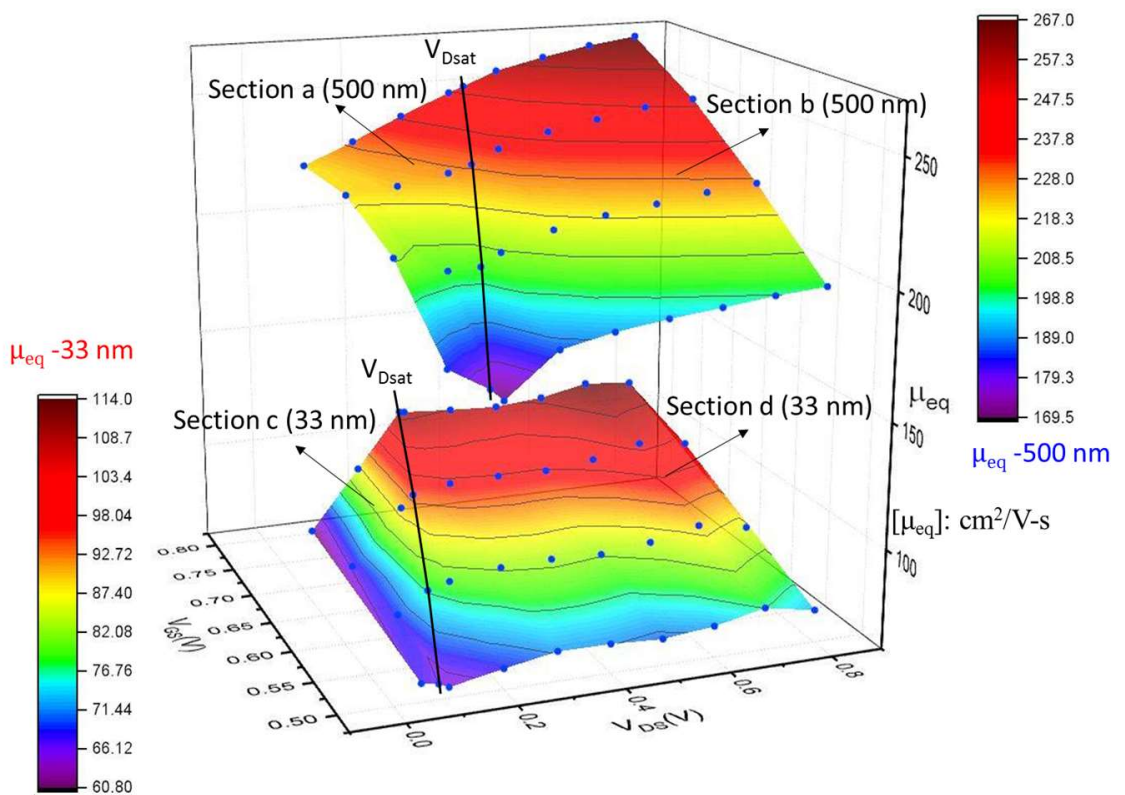

Fig. 4. Three dimensions of $\mu_{\mathrm{eq}}$ versus $\mathrm{V}_{\mathrm{DS}}$ and $\mathrm{V}_{\mathrm{GS}}$ at $\mathrm{L}_{\text {mask }}=500 \mathrm{~nm}$ (long-channel) and $33 \mathrm{~nm}$ (shortchannel).

Table III. The values of coefficients in section a under $\mathrm{V}_{\mathrm{GS}}=0.8 \mathrm{~V}$ and $\mathrm{L}_{\text {mask }}=500 \mathrm{~nm}$.

\begin{tabular}{ll}
\hline The value of $\mathrm{a}_{3}$ & $\mathrm{a}_{3}=-247.999$ \\
& $\mathrm{a}_{33}=19833.33$ \\
& $\mathrm{a}_{32}=-5150$ \\
& $\mathrm{a}_{31}=-29383.3$ \\
& $\mathrm{a}_{30}=16400$ \\
& \\
The values of $\mathrm{a}_{3 j} \mathrm{a} 32=-3283858.592$ \\
\\
$\mathrm{a} 331=5452922.151$ \\
$\mathrm{a} 330=-1885663.094$ \\
$\mathrm{a} 322=6422679$ \\
& $\mathrm{a} 321=-10725100.45$ \\
& $\mathrm{a} 320=3751730.477$ \\
& $\mathrm{a} 312=-4094362.073$ \\
& $\mathrm{a} 311=6889789.134$ \\
& $\mathrm{a} 310=-2450687.382$ \\
& $\mathrm{a} 302=840872.7189$ \\
The values of $\mathrm{a}_{3 j \mathrm{j}}$ & $\mathrm{a} 301=-1430708.191$ \\
& $\mathrm{a} 300=521535.9158$ \\
\hline
\end{tabular}

\section{IV. CONCLUSION}

Without considering the effect of channel length modulation and drift velocity overshoot, the mixed current model, including surface drift and diffusion current, has been announced to describe the electrical behavior of drive current of nano-node MOSFETs. The existence of diffusion current addresses why the drive current enters the saturation region, but not zero at the pinch-off point. The diffusion contribution in drive current becomes more apparent, especially for short-channel MOSFETs. For devices in normal operation, the 
parameters $\left(\mathrm{V}_{\mathrm{DS}}, \mathrm{V}_{\mathrm{GS}}\right.$, and $\left.\mathrm{L}_{\text {mask }}\right)$ independent of channel poistion are strongly correlated to the $\mu_{\text {eq }}$ value. Investigating the mutual interaction among them is indeed necessary. This work successfully provides the physical and meaningful consequences for nano-node MOSFET devices, fabricated with the ALD technology. The error between simulation and experimental results is less than $1 \%$, which is more suitable to the $28 \mathrm{~nm}$ devices or beyond to build up a more accurate set of device model in circuit design consideration.

\section{ACKNOWLEDGEMENT}

This work was supported in part by the Chung-Shan Institute of Science and Technology in Taiwan under Grant NCSIST-1164-V101 (106). The authors sincerely thank UMC in Taiwan provides the great 28-nm node logic wafers in this research.

\section{REFERENCES}

[1] B.G. Streetman, S.K. Banerjee, Solid state electronic devices. $7^{\text {th }}$ ed., Pearson, New Jersey, USA, 2016, pp.290-350.

[2] C. Hu, Modern semiconductor devices for integrated circuits. $1^{\text {st }}$ ed., Pearson, New Jersey, USA, 2010, pp.213-265.

[3] C. Nguyen-Duc, S. Cristoloveanu, G. Ghibaudo, "A three-piece model of channel length modulation in submicrometer MOSFETs," Solid-State Electronics, vol.31, iss.6, pp.1057-1063, 1988.

[4] K.Y. Lim, X. Zhou, "An analytical effective channel-length modulation model for velocity overshoot in submicron MOSFETs based on energy-balance formulation," Microelectronics Reliability, vol.42, iss.12, pp.1857-1864, 2002.

[5] B.J. Moon, C.K. Park, K. Lee, M. Shur, "New short-channel n-MOSFET current-voltage model in strong inversion and unified parameter extraction method," IEEE Trans. Electron Dev., vol.38, iss.3, pp.592-602, 1991.

[6] G.G. Shasidl, D. A. Antoniadis, H. I. Smith, "Electron velocity overshoot at room and liquid nitrogen temperatures in silicon inversion layers," IEEE Electron Dev. Lett., vol.9, iss.2, pp.94-96, 1988.

[7] K. K. Thornber, "Current equations for velocity overshoot," IEEE Electron Dev. Lett., vol.3, iss.3, pp.69-71, 1982.

[8] T. Kobayashi, K. Saito, "Two-dimensional analysis of velocity overshoot effects in ultrashort-channel Si MOSFET's," IEEE Trans. Electron Dev., vol.32, iss.4, pp.788-792, 1985.

[9] S. Y. Chou, D. A. Antoniadis, H. I. Smith, "Observation of electron velocity overshoot in sub-100-nm-channel MOSFET's in silicon," IEEE Electron Dev. Lett., vol.6, iss.12, pp.665-667, 1985.

[10] H.S. Huang, W.L. Wang, M.C. Wang, Y.H. Chao, S.J. Wang, S.Y. Chen, "I-V model of nano nMOSFETs incorporating drift and diffusion current," Vacuum, vol.155, pp.76-82, 2018. 